\title{
Insulin sensitivity is normalized in the third generation (F3) offspring of developmentally programmed insulin resistant (F2) rats fed an energy-restricted diet
}

\author{
Daniel C Benyshek*1, Carol S Johnston², John F Martin ${ }^{3}$ and \\ William D Ross ${ }^{4}$
}

Address: ${ }^{1}$ Department of Anthropology, University of Nevada Las Vegas, 4505 Maryland, Parkway, Box 455003, Las Vegas, NV 89154-5003, USA, ${ }^{2}$ Department of Nutrition, Arizona State University, Mesa, AZ, USA, ${ }^{3}$ School of Human Evolution and Social Change, Arizona State University, Tempe, AZ, USA and ${ }^{4}$ College of Medicine, University of Arizona, Tucson, AZ, USA

Email: Daniel C Benyshek* - daniel.benyshek@unlv.edu; Carol S Johnston - carol.johnston@asu.edu; John F Martin - john.martin@asu.edu; William D Ross - wross@email.arizona.edu

* Corresponding author

Published: 17 October 2008

Nutrition \& Metabolism 2008, 5:26 doi:10.1186/1743-7075-5-26
Received: 18 June 2008

Accepted: 17 October 2008

This article is available from: http://www.nutritionandmetabolism.com/content/5/1/26

(C) 2008 Benyshek et al; licensee BioMed Central Ltd.

This is an Open Access article distributed under the terms of the Creative Commons Attribution License (http://creativecommons.org/licenses/by/2.0), which permits unrestricted use, distribution, and reproduction in any medium, provided the original work is properly cited.

\begin{abstract}
Background/Aims: The offspring and grandoffspring of female rats fed low protein diets during pregnancy and lactation, but fed nutritionally adequate diets thereafter, have been shown to exhibit altered insulin sensitivity in adulthood. The current study investigates the insulin sensitivity of the offspring and grandoffspring of female rats fed low protein diets during pregnancy, and then maintained on energy-restricted diets post weaning over three generations.

Methods: Female Sprague Dawley rats (F0) were mated with control males and protein malnourished during pregnancy/lactation. FI offspring were then weaned to adequate but energyrestricted diets into adulthood. FI dams were fed energy-restricted diets throughout pregnancy/ lactation. F2 offspring were also fed energy-restricted diets post weaning. F2 pregnant dams were maintained as described above. Their F3 offspring were split into two groups; one was maintained on the energy-restricted diet, the other was maintained on an adequate diet consumed ad libitum post weaning.

Results: F2 animals fed energy-restricted diets were insulin resistant $(P<0.05)$, while the insulin sensitivity of their $\mathrm{F} 3$ offspring equaled and surpassed that of controls on both the energy-restricted and adequate ad libitum postweaning diets $(p<0.05)$.

Conclusion: Maternal energy-restriction did not consistently program reduced insulin sensitivity in offspring over three consecutive generations. The reasons for this remain unclear. It is possible that the intergenerational transmission of developmentally programmed insulin resistance is determined in part by the relative insulin sensitivity of the mother during pregnancy/lactation.
\end{abstract}

\section{Background}

An increasing number of experimental animal studies have demonstrated the intergenerational effects of devel- opmental programming on a variety of phenotypic traits, including birth weight, blood pressure, and glucose metabolism. While the investigation of the multigenera- 
tional effects of developmentally programmed traits is widening, very few studies have explored the potential for these traits to be transmitted beyond the second (F2) generation, or with post weaning diets other than adequatecontrol [1]. A recent study reported that glucose metabolism is altered in the adequately-nourished offspring (F1 through F3 generation) of dams (F0) malnourished during gestation and lactation [2]. Here we extend those findings with a pilot study examining the intergenerational effects of energy-restricted diets on developmentally programmed insulin sensitivity. As in previous studies, we found that the adult offspring (F1) of normal dams (F0) fed a low protein diet while pregnant and nursing were low birth weight and insulin sensitive, but that their adult offspring (F2) were less insulin sensitive than control animals. These animals (F1 and F2 generations) were maintained on a nutritionally adequate (i.e., standard 'control' chow) but energy-restricted diet (70\% of control ad libitum consumption) throughout life. However, the F3 generation, showed normal - and even enhanced insulin sensitivity - regardless of whether they were fed the same nutritionally-adequate but energy-restricted diet, or given ad libitum access to the nutritionally adequate diet post weaning.

\section{Materials and methods}

Sprague Dawley rats consumed either a nutritionally adequate diet (20\% protein; TD 91352, Harlan Teklad, Madison, $\mathrm{WI}$ ), or an isocaloric, low protein diet ( $8 \%$ protein; TD 93033, Harlan Teklad, Madison, WI) from day one of pregnancy through lactation. Litters were culled to 8 pups/ litter after birth weights were recorded. Pups whose mothers were protein malnourished in utero consumed an adequate but energy-restricted $(70 \%$ of ad libitum control daily consumption) diet postweaning. The dams of control pups were adequately nourished throughout pregnancy and their offspring consumed an adequate diet ad libitum postweaning. (An F2 generation of control animals was also bred, with no statistically significant differences observed between F1 and F2 control animals with respect to any body weight or metabolic measures.) At $~ 75$ days of age control and experimental animals were food deprived overnight and fasting glucose and insulin levels were measured. Animals were sacrificed under $\mathrm{CO} 2$ anesthesia. Blood was collected by cardiac puncture. Four female median body-weight first-generation (F1) rats whose mothers were protein malnourished during pregnancy and while nursing were selected from the experimental group at $\sim 75$ days of age, mated with control breeder males, and maintained on the energy-restricted diet throughout gestation and lactation. Their offspring, the F2 generation, also consumed a nutritionally adequate but energy-restricted diet post-weaning. At 75 days of age fasting glucose and insulin values were obtained from the F2 rats as described. A final generation of animals (F3) was bred from control breeder males and four median body-weight F2 dams whose grandmothers (F0) had been protein malnourished throughout pregnancy and lactation. F3 animals were then split into two groups. One group was maintained on the energy-restricted diet, while the other was given ad libitum access to the adequate diet post weaning. At $\sim 75$ days of age, all F3 animals were tested as described (Table 1). This research was approval by the Institutional Animal Care and Use Committee (IACUC) at Arizona State University and the University of Nevada Las Vegas. Animals were maintained in accordance with the 'Guide for the Care and Use of Laboratory Animals' of the National Research Council (7th ed., 1996).

\section{Results}

As in previous reports [1,3], the mean birth weight of both male and female F1 animals in our study were significantly lower $(\mathrm{p}<0.05)$ than control animals (Table 2$)$. Mean birth weights of F2 and F3 male and female animals did not differ significantly from control animals, although the mean weaning and 75 day weights were both signifi-

Table I: Research Design

\begin{tabular}{lll}
\hline Generation & Breeding Dam Prenatal/Nursing Diet & Offspring Post weaning Diet \\
\hline F0 & Low Protein & Energy-Restricted \\
\hline F1 & Energy-Restricted & Energy-Restricted \\
\hline F2 & Energy-Restricted & Energy-Restricted \\
\hline F3 & Energy-Restricted & F3.I Energy-Restricted
\end{tabular}

F3.2 Nutritionally-Adequate

Controls Nutritionally-Adequate Nutritionally-Adequate

Nutritionally Adequate diet - ad libitum (20\% protein; TD 91352, Harlan Teklad, Madison, WI); Low Protein diet - ad libitum (isocaloric, $8 \%$ protein; TD 93033, Harlan Teklad, Madison, WI); Energy-Restricted - 70\% control animal consumption of Nutritionally Adequate diet. 
cantly lower than those of controls animals, respectively. Our results (Figure 1) also show that the effects of maternal protein malnutrition during prenatal and suckling periods on glucose homeostasis of F1 and F2 offspring are consistent with previous studies where offspring were weaned onto adequate (control) diets: normal insulin sensitivity in F1 animals [4,5], and hyperinsulinemia/ insulin resistance in F2 animals [2,3]. Unexpectedly, our results also showed that the adult F3 offspring of insulin resistant F2 dams fed an energy-restricted diet during pregnancy and lactation and maintained on the restricted diet post weaning (F3.1), were equally insulin sensitive (females), or more insulin sensitive (males), than control animals, as measured by Homeostatic model assessment (HOMA). The insulin sensitivity of male and female experimental F3 offspring given ad libitum access to adequate diets post weaning (F3.2) improved to that of controls animals. While the general trends of intergenerational changes in glucose-metabolism are similar for both F1 - F3 males and females, the differences are more pronounced for females. Among F2 females, mean HOMA, insulin and glucose levels are all significantly ( $\mathrm{p}$ $<0.05$ ) higher than control values, with HOMA and insulin values over 250 and 200 percent, respectively, compared to controls. Among both post weaning diet groups of adult F3 females (F3.1, F3.2), however, HOMA, glucose
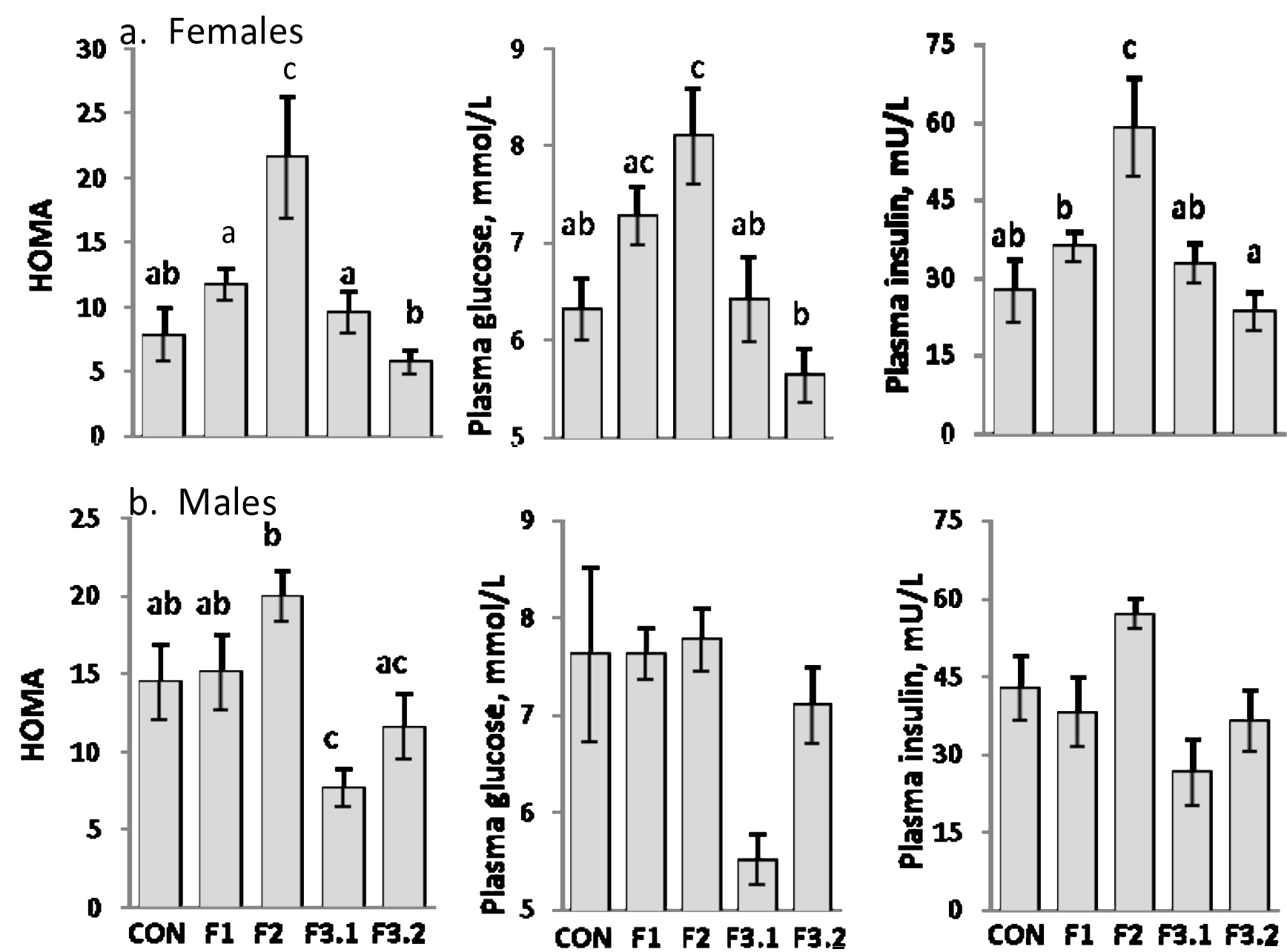

Figure I

HOMA, Fasting Glucose, and Fasting Insulin. HOMA, fasting glucose, and fasting insulin for female (a.) and male (b.) control rats (CON); rats whose dams consumed low-protein diets while pregnant and nursing (FI), offspring of FI rats (F2); and offspring of F2 rats (F3.I and F3.2). Control rat dams consumed adequate diets ad libitum during pregnancy and while nursing, and control offspring consumed adequate diets ad libitum postweaning. All FI, F2 and F3 offspring were fed nutritionally adequate but energy-restricted diets (70\% of control ad libitum consumption) postweaning with the exception of the F3.2 offspring that were fed the control diet ad libitum. Bars with different letters differ significantly $(p<0.05$, significant univariate ANOVA with LSD post-hoc analysis). 
Table 2: Birth Weight, Weaning Weight, and Adult Weight

\begin{tabular}{llll}
\hline Males & Birth Weight (g) & Weaning Weight (g) & 75 d Weight (g) \\
\hline Controls (10) & $7.1 \pm 0.4 \mathrm{a}$ & $68.7 \pm 1.4 \mathrm{a}$ & $449.0 \pm 8.8 \mathrm{a}$ \\
\hline FI (7) & $5.1 \pm 0.1 \mathrm{~b}$ & $23.9 \pm 1.7 \mathrm{~b}$ & $257.4 \pm 11.5 \mathrm{~b}$ \\
\hline F2 (7) & $6.6 \pm 0.2 \mathrm{ac}$ & $29.0 \pm 0.8 \mathrm{c} \pm 16.2 \mathrm{~b}$ \\
\hline F3.1 (7) & $5.8 \pm 0.2 \mathrm{bc}$ & $29.1 \pm 0.9 \mathrm{c}$ & $250.0 \pm 6.5 \mathrm{~b}$ \\
\hline F3.2 (7) & $6.6 \pm 0.3 \mathrm{ac}$ & $31.9 \pm 0.8 \mathrm{c}$ & $337.0 \pm 9.4 \mathrm{c}$ \\
\hline Females & & & $261.3 \pm 3.2 \mathrm{a}$ \\
\hline Controls $(3)$ & & $60.0 \pm 1.7 \mathrm{a}$ & $173.7 \pm 7.1 \mathrm{~b}$ \\
\hline FI (7) & $7.7 \pm 0.9 \mathrm{a}$ & $26.3 \pm 1.0 \mathrm{bc}$ & $187.7 \pm 8.8 \mathrm{~b}$ \\
\hline F2 (3) & $5.3 \pm 0.3 \mathrm{~b}$ & $29.3 \pm 0.3 \mathrm{c}$ & $182.8 \pm 5.5 \mathrm{~b}$ \\
\hline F3.1 (6) & $7.0 \pm 0.0 \mathrm{ac}$ & $26.0 \pm 0.9 \mathrm{~b}$ & $223.6 \pm 2.3 \mathrm{c}$ \\
\hline F3.2 (5) & $6.0 \pm 0.3 \mathrm{bc}$ & $28.8 \pm 0.7 \mathrm{c}$ & \\
\hline
\end{tabular}

Mean birth weights, $14 \mathrm{~d}$ weaning weights, and $75 \mathrm{~d}$ weights for control rats and rats whose dams consumed low-protein diets while pregnant and nursing (FI), offspring of FI rats (F2); and, offspring of F2 rats (F3.I and F3.2). Control rat dams consumed adequate diets ad libitum during pregnancy and while nursing, and control offspring consumed adequate diets ad libitum postweaning. All FI, F2 and F3 offspring were fed nutritionally adequate but energy-restricted diets (70\% of control ad libitum consumption) postweaning with the exception of the F3.2 offspring that were fed the control diet ad libitum. (Sample size shown in parentheses.) Means ( \pm SE) in columns with different letters differ significantly ( $<<0.05$, significant univariate ANOVA with LSD post-hoc analysis).

and insulin levels all effectively return to values of adequately fed control animals. Insulin sensitivity, as measured by HOMA, also improved dramatically among F3 males fed an energy-restricted diet post weaning (F3.1) relative to F2 males, and actually exceeds the insulin sensitivity of control animals $(\mathrm{p}<0.05)$. Insulin sensitivity of experimental F3 males given ad libitum access to an adequate diet post weaning (F3.2) also improved relative to F2 males, but improves only to that of control animals.

\section{Discussion}

The results from previous animal studies have shown that nutritional insults during pregnancy and lactation are linked to glucose intolerance via either hyperinsulinemia/ insulin resistance, or decreased insulin secretory capacity, depending on the type and timing of the prenatal nutritional insult. Maternal low-protein diets during pregnancy followed by control diets post weaning $[2,5]$, in addition to energy restricted prenatal diets during the latter half of pregnancy followed by control diets post weaning [6,7] are associated with insulin-sensitive offspring, but with reduced insulin secretory capacity. The findings reported here for F1 animals are consistent with the maternal protein malnutrition animal model that are linked with impaired glucose tolerance among the mature offspring - but without accompanying hyperinsulinemia/ insulin resistance. In contrast, other previous single-generation animal studies have shown that maternal energyrestricted diets fed throughout pregnancy lead to hyperinsulinemia/insulin resistance in adult offspring when followed by adequate and hypercaloric diets post weaning $[8,9]$. Similarly, we report here that reduced insulin sensitivity and hyperinsulinemia are also evident in (F2) animals whose mothers were fed energy-restricted diets throughout pregnancy/lactation and are then maintained on the same energy restricted diet post weaning. Why insulin sensitivity normalizes in the F3 offspring of such developmentally-programmed insulin resistant F2 females, however, even though they were exposed to the same prenatal and post weaning dietary protocols as their mothers (energy-restricted/energy-restricted) remains to be determined. This is especially puzzling given that insulin resistance is generally characterized as an 'adaptive' phenotypic trait in nutrient poor environments [10]. Based on the results presented here, it is possible that the insulin sensitivity of the mother fed an energy-restricted diet during pregnancy is a critical variable (i.e., offspring of insulin-resistant mothers fed energy-restricted diets develop normal insulin sensitivity, while the offspring of insulin-sensitive mothers fed energy-restricted diets dur- 
ing pregnancy become insulin resistant). In the current study, the reason for this may be that the maternal insulin resistance of pregnant dams fed an energy-restricted diet increases the availability of nutrients for the fetus - in effect normalizing fetal nutrition and development while the relative insulin sensitivity of pregnant dams fed an energy-restricted diet makes fewer nutrients available for the developing fetus and programs for insulin resistance in the offspring. For F3 animals whose (F2) mothers were fed energy-restricted diets during pregnancy/lactation, but that were fed adequate diets ad libitum post weaning (F3.2), their improved insulin sensitivity, despite significant $(\mathrm{p}<0.05)$ increases in adult body weight, suggests that maternal diet during gestation/lactation, and not post weaning diet or body weight is the critical variable determining relative insulin sensitivity/resistance. This is of particular interest, given that our group has previously shown that the adequately-nourished grand-offspring (F3) of (F1) rats malnourished during gestation and perinatal life remain significantly less insulin sensitive than control animals [2], suggesting that it is the energy-restriction during pregnancy and lactation in the current study that results in the improved insulin sensitivity of F3 animals. Sex differences are evident in the data in that changes in glucose-insulin metabolism were more pronounced, and more often reached the level of statistical significance, among females than among males. The overall pattern of intergenerational change in glucosemetabolism is similar for both F1 - F3 males and females, however, and among both sexes, insulin resistance (as measured by HOMA), along with fasting insulin and glucose levels, peak in the F2 generation. This leaves the extent of the reduction of insulin resistance between F3.1 (energy restricted post weaning diet) and F3.2 (ad libitum post weaning diet) male and female animals as the only other significant sex-based difference. Here, among both males and females, insulin sensitivity improves dramatically among all F3 animals (whose mothers were fed an energy restricted diet during pregnancy), but among females the larger improvement is among the F3.2 (ad libitum) post weaning diet; whereas among males, insulin sensitivity is most improved among animals fed the F2.1 (energy-restricted post weaning) diet. These sex-based differences should be interpreted with caution, however, because while differences exist in the degree of improved insulin sensitivity between F3 males and females by post weaning diet, among F3.1 and F3.2 females HOMA values do not differ significantly from female controls; and HOMA values of F3.1 and F3.2 males do not differ significantly from each other.

\section{Conclusion}

Contrary to findings in previous studies in which the offspring and grandoffspring of female rats fed low protein diets during pregnancy and lactation, but fed nutritionally adequate diets thereafter, exhibit altered insulin sensitivity in adulthood [2], the current study found that maternal energy-restricted diets during pregnancy and nursing, followed by post weaning energy restricted diets, did not consistently program reduced insulin sensitivity among F2 and F3 offspring of maternally protein malnourished dams (F1). Instead, F2 animals were hyperinsulinemic/ insulin resistant, while F3 animals subjected to the identical diets throughout their life course were at least as insulin sensitive as control animals. The reasons for this remain unclear. It is possible that the intergenerational transmission of developmentally programmed insulin resistance is determined in part by the relative insulin sensitivity of the mother during pregnancy/lactation. Further research is needed on the metabolic effects of pre and postnatal diets, varying in nutrient composition and over multiple generations, in order to better understand the fundamental principles of the transgenerational transmission of developmentally programmed traits. Such further investigation of the variability in the intergenerational transmission of developmentally programmed traits is clearly warranted, not only as a matter of etiological significance, but for its relevance to possible future disease prevention applications among humans.

\section{Competing interests}

The authors declare that they have no competing interests.

\section{Authors' contributions}

DCB designed the study, collected assay blood samples, and drafted the manuscript. CSJ collected assay blood samples, and performed the statistical analysis and helped draft the manuscript. JFM contributed to study design and data analysis. WDR helped with the study design, collected assay blood samples, and was responsible for daily animal husbandry. All authors read and approved the final manuscript.

\section{Acknowledgements}

The authors would like to thank Mike Stroup (ASU Nutrition Laboratory) for performing all of the laboratory assays.

This research was funded by a research incentive award from the Division of Research and Graduate Studies at the University of Nevada Las Vegas.

\section{References}

I. Drake AJ, Walker BR: The intergenerational effects of fetal programming: non-genomic mechanisms for the inheritance of low birth weight and cardiovascular risk. J Endocrinol 2004, 180:1-16.

2. Benyshek DC, Johnston CS, Martin JF: Glucose metabolism is altered in the adequately-nourished grand-offspring (F3 generation) of rats malnourished during gestation and perinatal life. Diabetologia 2006, 49(5): I I 17-1 II9.

3. Ozanne S: Metabolic programming in animals. Br Med Bull 200I, 60:143-152.

4. Martin JF, Johnston CS, Han C-T, Benyshek DC: Nutritional origins of insulin resistance: a rat model for diabetes-prone human populations. J Nutr 2000, 130:74I-4. 
5. Dahri S, Snoeck A, Reusens-Billen B, Remacle C, Hoet J]: Islet function in offspring of mothers on low-protein diet during gestation. Diabetes I99I, 40(suppl 2): I I5-I 20.

6. Garafano A, Czernichow P, Breant B: In utero undernutrition impairs rat beta-cell development. Diabetologia 1997, 40:1231-1234

7. Holemans K, Gerber R, Meurrens K, De Clerck F, Poston L, Van Assche FA: Maternal food restriction in the second half of pregnancy affects vascular function but not blood pressure of rat female offspring. Br J Nutr 8I:73-79.

8. Vickers MH, Breier BH, Cutfield WS, Hofman PL, Gluckman PD: Fetal origins of hyperphagia, obesity, and hypertension and postnatal amplification by hypercaloric nutrition. Am J Physiol Endocrinol Metab 2000, 279:E83-E87.

9. Kind KL, Clifton PM, Grant PA, Owens PC, Sohlstrom A, Roberts CT, Robinson JS, Owens JA: Effect of maternal feed restriction during pregnancy on glucose tolerance in the adult guinea pig. Am J Physiol Regul Integr Comp Physiol 2003, 284:R I 40-R I 52.

10. Gluckman P, Hanson M, Beedle A: Early life events and their consequences for later disease: a life history and evolutionary perspective. Am J Hum Biol 2007, 19:1-19.

Publish with Bio Med Central and every scientist can read your work free of charge

"BioMed Central will be the most significant development for disseminating the results of biomedical research in our lifetime. "

Sir Paul Nurse, Cancer Research UK

Your research papers will be:

- available free of charge to the entire biomedical community

- peer reviewed and published immediately upon acceptance

- cited in PubMed and archived on PubMed Central

- yours - you keep the copyright

Submit your manuscript here:

http://www.biomedcentral.com/info/publishing_adv.asp 